\title{
Computer Network Information Security and Protection Measures
}

\author{
Qingbao Meng \\ Tianjin Maritime College(China Tianjin300350)
}

Keywords: Network security; Computer; Threat; Protective measures

\begin{abstract}
With the rapid development of science and technology, the computer has been widely used in people's life and work in all aspects with bringing convenience to people's lives at the same time, and it is also gradually changing people's way of life, such as computer office, email, video chat and so on. However, the ensuing network security problems also pose a great threat to people's life and work. Therefore, how to ensure the information security of computer network has become the main problem faced by people, this paper mainly discusses and researches on the security of computer network information and protection measures to provide theoretical support for the computer network information security.

Since twenty-first Century, the world economy has been developing rapidly. As a new technical means, computer network technology has been widely used in all aspects of people's life and work. The computer network technology brings convenience to people's life and work, at the same time, it has brought great hidden dangers to people's information security, and some hackers have a bottom line and prying privacy problems caused by the leakage of personal information is not in every hour and moment threatening people's personal and property safety. Therefore, to find out the security problems existing in the computer network, and then find out the appropriate measures to solve the problems is the most effective way to solve the network security problems.
\end{abstract}

\section{Part One Common problems in computer network information security}

\section{Natural disaster}

The computer is a semi intelligent machine which is able to complete some operations established according to the instruction of people, such as most machines, various external reasons or natural disasters that affected operating conditions in the process of computers. For example, damp room environment, vibration or voltage stability would affect the status of the computer, so the developed countries in the world are specifically for some operating computer center or large computer to do the corresponding proof, lightning protection and voltage stabilizing device to avoid the harm of natural disasters for computer.

\section{Network systems are vulnerable to attacks}

The era is a breakthrough in time and space of network technology, because of its openness, it has brought great convenience to people's life and work, but it is also because of its openness and the computer is vulnerable to get attacks from the network. And computer network's worldwide operations need to rely on some simple IP protocol, due to their poor safety performance, the network protocol lead to the computer network system that will be a series of network security problems of network attacks, data loss and so on.

\section{User's improper operation and hacker attack}

Due to the rapid development of China's economy, the popularity of computer network in China people is also very high, and the Chinese people's security awareness for the computer network is not in place but also resulted in China's computer network information security risk. Such as people in the computer network operation, because of their computer network security awareness is weak and unconscious disclosure of their accounts or passwords. While the computer network security problem is hacker, and hacker attacks mainly refers to the unconventional methods of malicious hacking into the computer, data copy or destroy the operation means of hacker attacks with various means, and the adverse consequences bring great harm for the country and people. 


\section{Part Two Computer network information security protection countermeasures}

\section{Strengthen people's awareness of network information security}

In view of China's current common network security problems such as bank account number and password loss caused by WeChat and QQ and other social software to account theft fraud and other frequently, China's education sector should be combined with the public security departments for the citizen network information security publicity and education work to prevent the increase of network security the consciousness of their own citizens, as citizens in the Internet and office conscious storage of documents, set the password, in addition to avoid as much as possible on the bank card payment password, ID card information and online payment passwords and other important information stored in the computer, only China's citizens consciously develop network information security awareness, then it will stop the occurrence of some network security incidents to a great extent.

\section{Installing antivirus and firewall software}

Due to the openness of computer network, there is a great threat to the security of computer network information. In order to minimize and avoid such harm, the network firewall technology is generally used. This technology mainly uses the limited network access to realize the computer network security control. Antivirus software is some of the common features of the virus writing software and anti-virus software in operation which will be designed for these features and virus killing, so as to ensure the safety of computer network by taking a side with computer anti-virus software and firewalls in our household and office are installed, and it needs regular of virus library in order to achieve real-time updates with the function of killing the virus.

\section{File encryption technology}

Due to the more time on current people's life and work with the computer, the resulting files can not be timely transferred to the mobile device, but usually stored in the computer, then it needs to consider the security of computer files by file encryption method. File encryption is mainly aimed at the file encryption in the file transmission and storage. File encryption mainly refers to the file transfer through the network, its principle is to upload files to the network, and then downloaded by the other one, and when the file transfer to the network, while if not timely file encryption, the file will inevitably lead to the leak. The file storage encryption refers to the use of public computers or computers do not set the landing password, you need to set file encryption to protect the security of the document. At present, file encryption technology is divided into by setting a password file for file encryption and digital signature encryption, the two encryption methods are through a series of complex mathematical algorithms to encrypt the file.

\section{IP address hiding}

Through the analysis and collation of network attacks, it is found that hackers mainly attack the computer by querying the IP address of the other party, and then attack the IP address. In a computer network, if the other party knows your IP address, then everything will be missed in other's view. At present, the common means to hide the IP address is the user through a proxy server for the operation of the Internet, because hackers will be unable to find the right IP, and the cannot bring remote operation and attack.

\section{Close unnecessary ports}

Because there are some useless computer ports in the computer system and network system, the hacker will invade the reserved port of the computer by scanning the scanner, and then invade the computer. In view of this situation, we can usually use a dedicated port to close the program or manually shut down some unnecessary ports, as far as possible to avoid hackers through computer network port intrusion.

\section{Protect administrator account and deny Guest account invasion}

For a computer, the administrator account has the highest authority to operate the computer, and hackers once master the computer administrator account, there will be a great security crisis. So in the computer operation, we can set the complex administrator account password to reduce the probability of hacking or by resetting the new administrator account, to hide the real administrator account in order to achieve the purpose of protecting the security of computer network. Guest 
account as a guest in the computer account administrator has not set permissions restrictions can access the computer directly, so set up a Guest account access or password access level is the main method to ensure the security of computer network access.

\section{Shut down common backdoor programs for hackers in computers}

The study finds that computer hackers often rely on the "back door": firstly, uninstall unnecessary network protocol for computer Internet, generally only need to implement the TCP/IP protocol on the line, and some others are not necessary agreement or even network security flaws such as NetBIOS, through the investigation and statistics after that, many network intrusion cases are implemented by using NetBIOS; secondly, close the file and print sharing, under normal circumstances, open the file and print sharing for our work are very convenient, but it is also easy to be hacked computer port, so that the user can open this button when in use, and close the button after use; thirdly, to prevent computer network port open, if without remote computer operation demand, the remote control button should be closed in time, which can improve the network speed of the computer and ensure the remote operation and intrusion of the computer in the process of operation.

\section{Preparing for safety Internet preparation}

With the wide application of computer network technology in our life and work, we must pay attention to the importance of network security, network security in the Internet with doing preparatory work before the current is a necessary mean to secure access to the Internet, preparations mainly include the following aspects: firstly, open the page in front. To do some restrictions and malicious code attack program that are ready to run on the browser, which set up the web page code in the browser and access to be selected with web address or trusted sites, as far as possible to avoid some of the code page attack; secondly, to open the security software necessary, we generally will make the software firewall and antivirus with the computer software that is set to start, so that we can ensure that the computer is protected in the Internet to avoid free computer in the Internet when attacked by hackers and endanger the documents and information security; thirdly, open anti Trojan program. Trojan refers to some illegal code disguised as normal file format stored in our computer, the computer will automatically run in the Internet, computer files and pass out copies. Therefore, to open the Trojan horse degree to scan the computer file to ensure the security in the Internet and computer files, and to regularly manually delete the registry all beginning with "Run" program files.

\section{Part Three Conclusion}

To sum up, there is a big difference between the speed of computer network application and the speed of computer network education in our country, which also leads to a series of problems of computer network security in a certain extent. This paper focuses on the computer network problems such as natural factors, safety awareness is weak, their computer viruses hackers are introduced in detail, and aiming at these problems, puts forward corresponding preventive measures such as strengthening people's awareness, install the virus killing and Internet security software, the implementation of file transfer and storage and encryption operation regular bug fixes and virus file delete work on the computer, and these are the ways to ensure the information security of the computer network, information, protection of property and personal safety.

\section{Reference}

[1]Guangwu Yang, Zhibo Zhao.Protection strategy of computer network information security[J].Digital World, 2017(5): 52.

[2]Guanxiong Wang.Computer network information security and coping strategies[J].Information and Computer (Theory Edition), 2017(1): 193-194.

[3]Yipin Chen.Computer network information security and its protection[J].Great Orient, 2017(6): 312. 